\title{
Limits to Arbitrage When Market Participation Is Restricted
}

Citation for published version (APA):

Hens, T., Herings, P. J. J., \& Predtetchinski, A. (2006). Limits to Arbitrage When Market Participation Is Restricted. Journal of Mathematical Economics, 42, 556-564.

https://doi.org/10.1016/j.jmateco.2006.04.001

Document status and date:

Published: 01/01/2006

DOI:

10.1016/j.jmateco.2006.04.001

Document Version:

Publisher's PDF, also known as Version of record

Document license:

Taverne

Please check the document version of this publication:

- A submitted manuscript is the version of the article upon submission and before peer-review. There can be important differences between the submitted version and the official published version of record.

People interested in the research are advised to contact the author for the final version of the publication, or visit the DOI to the publisher's website.

- The final author version and the galley proof are versions of the publication after peer review.

- The final published version features the final layout of the paper including the volume, issue and page numbers.

Link to publication

\footnotetext{
General rights rights.

- You may freely distribute the URL identifying the publication in the public portal. please follow below link for the End User Agreement:

www.umlib.nl/taverne-license

Take down policy

If you believe that this document breaches copyright please contact us at:

repository@maastrichtuniversity.nl

providing details and we will investigate your claim.
}

Copyright and moral rights for the publications made accessible in the public portal are retained by the authors and/or other copyright owners and it is a condition of accessing publications that users recognise and abide by the legal requirements associated with these

- Users may download and print one copy of any publication from the public portal for the purpose of private study or research.

- You may not further distribute the material or use it for any profit-making activity or commercial gain

If the publication is distributed under the terms of Article $25 \mathrm{fa}$ of the Dutch Copyright Act, indicated by the "Taverne" license above, 


\title{
Limits to arbitrage when market participation is restricted
}

\author{
Thorsten Hens ${ }^{\text {a }}$, P. Jean-Jacques Herings ${ }^{b}$, Arkadi Predtetchinskii ${ }^{b}$,* \\ a Institute for Empirical Research in Economics, University of Zurich, Blümlisalpstr. 10, 8006 Zürich, Switzerland \\ ${ }^{\mathrm{b}}$ Department of Economics, Universiteit Maastricht, P.O. Box 616, 6200 MD Maastricht, The Netherlands
}

Received 21 December 2005; accepted 12 April 2006

Available online 30 May 2006

\begin{abstract}
There is an extensive literature claiming that it is often difficult to make use of arbitrage opportunities in financial markets. This paper provides a new reason why existing arbitrage opportunities might not be seized. We consider a world with short-lived securities, no short-selling constraints and no transaction costs. We show that to exploit all existing arbitrage opportunities, traders should pay attention to all financial markets simultaneously. The paper gives a general result stating that failure to do so will leave some arbitrage opportunities unexploited with probability one.

(C) 2006 Elsevier B.V. All rights reserved.
\end{abstract}

JEL classification: D52; G12

Keywords: Arbitrage; Bounded rationality

\section{Introduction}

One of the fundamental concepts in finance is arbitrage, defined as the simultaneous purchase and sale of the same, or essentially similar, security in two different markets for advantageously different prices, see Sharpe and Alexander (1990). The efficient market hypothesis relies to a large extent on the assumption that, whenever present, arbitrage opportunities will be exploited quickly. The behavioral finance literature as in Shleifer (2000, p. 2), questions this hypothesis:

\footnotetext{
* Corresponding author. Tel.: +31 43388 3906; fax: +31 433884878.

E-mail addresses: thens@iew.unizh.ch (T. Hens), P.Herings@algec.unimaas.nl (P. Jean-Jacques Herings), A.Predtetchinski@algec.unimaas.nl (A. Predtetchinskii).
} 
The key forces by which markets are supposed to attain efficiency, such as arbitrage, are likely to be much weaker and more limited than the efficient markets theorists have supposed.

In reality arbitrage opportunities are limited by a number of factors like the existence of transactions costs, short-selling contraints, or mispricing of securities deepening in the short run.

This paper claims that even under close to ideal circumstances, i.e. the case where transactions costs are zero, short-selling constraints do not exist and securities are short-lived (so that deepening of mispricing is impossible), existing arbitrage opportunities might not be seized. We show that this is generally the case whenever traders restrict their attention to a subset of the securities traded at a certain point in time.

Following Radner and Rothschild (1975), many authors have observed that attention is only available in limited amounts. Van Zandt (1999) argues that individuals are bounded not so much by the total amount of information processing they can handle, as by the amount they can perform in a given amount of time. This leads to parallel or distributed processing, where information processing tasks are broken down into steps that are shared among the members of the organization and where each of these steps takes time.

Limits to the capability of information processing are the main reasons for traders do specialize to subsets of securities. In investment firms, for instance, analysts typically concentrate on the stocks within a particular industry sector. In Vayanos (2003), this feature is modeled by a processing constraint. Agents are assumed to analyze portfolios of at most a fixed number of inputs, where an input can either be an asset examined directly, or a subordinate's portfolio.

In this paper we incorporate information processing constraints in the finance version of the general equilibrium model with incomplete asset markets. The model is a special case of the restricted market participation models of Siconolfi (1988) and Polemarchakis and Siconolfi (1997). Since we assume a two-period time horizon, all traded assets are short-lived. This is the most favorable case for absence of arbitrage, as it makes deepening of mispricing impossible. Investors can buy and sell assets in period 0 without being subject to short-selling constraints or transactions costs. They are however subject to information processing constraints. An investor is assumed to be unable to be active in the markets of all traded assets simultaneously.

Assets have payoffs in period 1, depending on the realization of the state of nature. Asset payoffs are real, i.e. denominated in terms of the consumption good. Investors consume in both periods. In this context, the usual definition of no-arbitrage is both the absence of a costless portfolio with non-negative returns in each future state of nature and strictly positive returns in at least one state, and of a portfolio yielding income in period 0 and with non-negative returns in each future state of nature.

Since investors restrict their attention to certain subsets of assets, they might not be able to make use of certain arbitrage opportunities. One might expect, however, that, under suitable assumptions, they are able to do so collectively. In particular, one might expect that this is the case as long as the subsets of assets to which investors pay attention overlap. This paper makes the point that this intuition is wrong. For almost all asset structures, as soon as each investor is limited in his trading opportunities to some extent, some arbitrage opportunities will be left unexploited, even at the collective level.

A related work in the literature is the one of Basak and Croitoru (2000), who also study the violation of no-arbitrage conditions. They consider an infinite horizon framework with three assets, a bond, a stock and a derivative. They put short-sales constraints on assets and upper bounds on the wealth that can be spent. We, on the other hand, exclude trade in some assets by some 
individuals altogether, but do not impose any transaction costs, and in particular no short-sales constraints, on assets individuals can trade in.

Section 2 outlines our model and derives the appropriate no-arbitrage conditions. Section 3 shows a first example that the no-arbitrage conditions in a restricted market participation model may differ from the usual no-arbitrage conditions. Section 4 derives the main result: this is typically the case, no matter how small the restriction in market participation.

\section{Arbitrage}

We consider the case that is most favorable to arbitrage. In particular, we consider a model with two time periods, $t=0,1$, and one state of nature $s$ out of $S$ possible states of nature realizing at $t=1$. At each time period, in each state of nature, there is a single good available, called income.

There is a finite number of investors $i=1, \ldots, I$. At $t=0$ investors allocate their money between consumption and investment in one of the available assets $j=1, \ldots, J$. Throughout we restrict attention to the case $J \leq S$. The symbols $\mathcal{I}, \mathcal{J}$, and $\mathcal{S}$ denote the sets $\{1, \ldots, I\},\{1, \ldots, J\}$, and $\{1, \ldots, S\}$, respectively.

Assets payoff in period 1 . The payoff of asset $j$ in state $s$ is given by $A_{s}^{j}$. Investor $i$ has a utility function $U^{i}$ and an initial income stream $\omega^{i} \in \mathbb{R}_{+}^{S+1}$. The set of possible income streams is given by $X^{i}=\mathbb{R}_{+}^{S+1}$.

Investor $i$ has only access to a limited set of asset markets. Cognitive restrictions require him to restrict attention to the set $\mathcal{J} \subset \mathcal{J}$ of assets. In reality, investors do indeed concentrate on a limited set of assets. The literature on information processing constraints explains why this is so.

Let $q \in \mathbb{R}^{J}$ denote the asset prices and $\theta^{i} \in \mathbb{R}^{J}$ the net asset portfolio of agent $i$, i.e. negative components of $\theta^{i}$ denote sales of the corresponding assets and positive components denote purchases.

The optimization problem of investor $i$ is given by

$$
\max _{\theta^{i} \in \mathbb{R}^{J}, x^{i} \in \mathbb{R}_{+}^{S+1}} U^{i}\left(x^{i}\right)
$$

subject to

$$
x^{i}-\omega^{i} \leq\left(\begin{array}{c}
-q \\
A
\end{array}\right) \theta^{i}, \quad \theta_{j}^{i}=0, \quad j \in \mathcal{J} \mathcal{J}^{j}
$$

Investor $i$ has arbitrage opportunities if he can purchase a portfolio at no cost today, with nonnegative payoffs at each state $s$ and a strictly positive payoff in at least one state, or if he can purchase a portfolio yielding positive income in period 0 and non-negative payoffs at each future state. For investor $i$ this leads to the following no-arbitrage condition, which is labelled NAC $i$ :

$$
\left(\mathrm{NAC}^{i}\right) \text { there is no } \theta^{i} \in \mathbb{R}^{J} \text { such that } \theta_{j}^{i}=0 \text { for all } j \in \mathcal{J} \mathcal{J}^{i} \text { and }\left(\begin{array}{c}
-q \\
A
\end{array}\right) \theta^{i} \in \mathbb{R}_{+}^{S+1} \backslash\{0\}
$$

It is well-known that $\mathrm{NAC}^{i}$ is satisfied if and only if $q \in Q^{i}$, where

$$
Q^{i}=\left\{q \in \mathbb{R}^{J} \mid \exists \pi \in \mathbb{R}_{++}^{S} \text { such that for every } j \in \mathcal{J}^{i}, q_{j}=\sum_{s \in \mathcal{S}} \pi_{s} A_{s}^{j}\right\}
$$


The following result is obvious

$$
\mathcal{J}^{i^{1}} \subset \mathcal{J}^{i^{2}} \Rightarrow Q^{i^{2}} \subset Q^{i^{1}}
$$

Asset prices are said to satisfy the no-arbitrage condition NAC if the no-arbitrage condition is satisfied for all investors. So asset prices $q$ satisfy NAC if and only if NAC ${ }^{i}$ is satisfied for every $i \in \mathcal{I}$. This is easily seen to be equivalent to the statement that $q \in \cap_{i \in \mathcal{I}} Q^{i}$.

\section{Networks of agents}

Another interesting no-arbitrage condition is the one which follows if some omniscient investor could oversee all the possibilities offered in the market. This leads to the market no-arbitrage condition $\mathrm{NAC}^{\mathrm{m}}$ :

$$
\left(\mathrm{NAC}^{\mathrm{m}}\right) \text { there is no } \theta \in \mathbb{R}^{J} \text { such that }\left(\begin{array}{c}
-q \\
A
\end{array}\right) \theta^{i} \in \mathbb{R}_{+}^{S+1} \backslash\{0\}
$$

It is well-known that $\mathrm{NAC}^{\mathrm{m}}$ is satisfied if and only if $q \in Q^{\mathrm{m}}$, where

$$
Q^{\mathrm{m}}=\left\{q \in \mathbb{R}^{J} \mid \exists \pi \in \mathbb{R}_{++}^{S} \text { such that } q=\sum_{s \in \mathcal{S}} \pi_{s} A_{s}\right\}
$$

The following proposition is easily shown.

Proposition 3.1. It holds that $Q^{\mathrm{m}} \subset \cap_{i \in \mathcal{I}} Q^{i}$.

Proposition 3.1 states that $\mathrm{NAC}^{\mathrm{m}}$ implies NAC. Of course, if some agent can trade in all markets, then the concepts of NAC and NAC ${ }^{\mathrm{m}}$ coincide.

Proposition 3.2. If for some investor $i \in \mathcal{I}$ it holds that $\mathcal{J}=\mathcal{J}$, then $Q^{\mathrm{m}}=\cap_{i \in \mathcal{I}} Q^{i}$.

Investor $i$ in the proposition is omniscient, so the result follows trivially.

A first intuition would be that if the $\mathcal{J}$ overlap, then NAC implies NAC ${ }^{\mathrm{m}}$. The market of asset $j^{\prime}$ is said to be related to the market of asset $j^{\prime \prime}$ if for some agent $i \in \mathcal{I}$ it holds that $j^{\prime}, j^{\prime \prime} \in \mathcal{J}$. The market of asset $j^{\prime}$ is said to be indirectly related to the market of asset $j^{\prime \prime}$ if there is a sequence of markets $j^{1}, \ldots, j^{n}$ such that $j^{1}=j^{\prime}$ and $j^{n}=j^{\prime \prime}$ and $j^{k}$ and $j^{k+1}$ are directly related for all $k \in\{1, \ldots$, $n-1\}$.

Neither direct relatedness nor indirect relatedness of all markets is sufficient for the sets $\cap_{i \in \mathcal{I}} Q^{i}$ and $Q^{\mathrm{m}}$ to coincide. In fact, consider the following example where it holds that all markets are directly related. Notice that one needs at least three assets for an interesting example.

Example 3.1. Suppose that $I=3, S=3, J=3$, and the matrix of asset payoffs is given by

$$
A=\left[\begin{array}{lll}
2 & 1 & 1 \\
1 & 2 & 1 \\
1 & 1 & 2
\end{array}\right]
$$

Assume that investor $i \in \mathcal{I}$ cannot trade in asset $i$. So, $\mathcal{J}^{1}=\{2,3\}, \mathcal{J}^{2}=\{1,3\}$ and $\mathcal{\mathcal { P }}=\{1,2\}$. Consider the asset price system $q=(5,3,5)$. We claim that $q \notin Q^{\mathrm{m}}$. Indeed, $\theta=(-1,+3,-1)$ is an arbitrage portfolio. However, $q$ belongs to $\cap_{i \in \mathcal{I}} Q^{i}$ because for $\mathcal{J}^{1}=\{2,3\}, \pi=(1 / 2,1 / 6$, $13 / 6)$, for $\mathcal{J}^{2}=\{1,3\}, \pi=(1,2,1)$ and for $\mathcal{J}^{3}=\{1,2\}, \pi=(13 / 6,1 / 6,1 / 2)$ are state price vectors demonstrating the absence of arbitrage opportunities. 
The example is the strongest example possible in the sense that adding one market to one agent gives equivalence between NAC and $\mathrm{NAC}^{\mathrm{m}}$ by Proposition 3.2.

\section{Limits to arbitrage}

This section shows that Example 3.1 is not an exceptional case. It makes the striking observation that in finance economies with restricted market participation, forgone arbitrage opportunities are the rule rather than the exception.

Let $\mathcal{A}$ denote the set of $(S \times J)$-dimensional matrices. Let $\mathcal{A}_{+}$be the set of those matrices in $\mathcal{A}$ for which there is a non-trivial asset portfolio giving non-negative returns in each state:

$$
\mathcal{A}_{+}=\left\{A \in \mathcal{A} \mid \text { there exists } \theta \in \mathbb{R}^{J} \backslash\{0\} \text { such that } A \theta \in \mathbb{R}_{+}^{S}\right\}
$$

We will restrict attention to the set of asset return matrices $\mathcal{A}_{+}$. Asset return matrices outside $\mathcal{A}_{+}$are hardly interesting: as is demonstrated in the next proposition, if the asset return matrix lies outside $\mathcal{A}_{+}$, then the conditions $\mathrm{NAC}^{i}$ and $\mathrm{NAC}^{\mathrm{m}}$ do not impose any restrictions on the asset prices.

Proposition 4.1. Let $A \in \mathcal{A}$. If $A$ is not in $\mathcal{A}_{+}$, then $Q^{i}=\mathbb{R}^{J}$ for all $i \in \mathcal{I}$ and $Q^{\mathrm{m}}=\mathbb{R}^{J}$. Conversely, if $Q^{\mathrm{m}}=\mathbb{R}^{J}$, then $A$ is not in $\mathcal{A}_{+}$.

Proof. The first part of the proposition is obvious: if $A$ is not in $\mathcal{A}_{+}$, then there is no nonzero portfolio of assets that gives a non-negative return in each state. Thus, by the definition of no-arbitrage, any price vector $q$ trivially satisfies conditions $\mathrm{NAC}^{i}$ and $\mathrm{NAC}^{\mathrm{m}}$.

To prove the converse, suppose there exists a price vector $q \in \mathbb{R}^{J} \backslash Q^{\mathrm{m}}$. Then, $\{\lambda q \mid \lambda>0\} \cap$ $\left\{\pi A \mid \pi \in \mathbb{R}_{++}^{S}\right\}$ is an empty set. By the separating hyperplane theorem, there exists $\theta \in \mathbb{R}^{J} \backslash\{0\}$ such that $\lambda q \theta \leq \pi A \theta$ for all $\lambda>0$ and $\pi \in \mathbb{R}_{++}^{S}$. Taking the limit for $\lambda \downarrow 0$, we obtain $0 \leq \pi A \theta$ for all $\pi \in \mathbb{R}_{++}^{S}$. For every $\sigma \in \mathcal{S}$, taking the limit $\pi_{\sigma} \rightarrow 1$ and $\pi_{s} \downarrow 0, s \in \mathcal{S} \backslash\{\sigma\}$, we get $0 \leq A_{\sigma} \theta$. Hence, $A \theta \in \mathbb{R}_{+}^{S}$, so $A \in \mathcal{A}_{+}$.

The next theorem is the main result of the paper. It claims that for a generic matrix in $\mathcal{A}_{+}$there is at least one price vector that satisfies the condition $\mathrm{NAC}^{i}$ for all $i$ but violates the condition $\mathrm{NAC}^{\mathrm{m}}$.

Theorem 4.1. Suppose that for all investors $i \in \mathcal{I}$ it holds that $\mathcal{J} \neq \mathcal{J}$. Then there exists an open subset $\mathcal{A}_{+}^{*}$ of the set $\mathcal{A}_{+}$with $\mathcal{A}_{+} \backslash \mathcal{A}_{+}^{*}$ having Lebesgue measure zero such that $Q^{\mathrm{m}} \neq \cap_{i \in \mathcal{I}} Q^{i}$ for all $A \in \mathcal{A}_{+}^{*}$.

The proof of Theorem 4.1 consists of two steps. Step one provides a necessary and sufficient condition for the coincidence of the sets $Q^{\mathrm{m}}$ and $\cap_{i \in \mathcal{I}} Q^{i}$ in terms of so-called "fundamental portfolios". Convex analysis is employed as the main tool in the first step of the proof. As a second step, we use the Transversality Theorem to demonstrate that this condition imposes too many restrictions on the asset return matrix.

Let $C$ be a closed convex cone. A half-line $L$ emanating from the origin is called an extreme ray of $C$ if $L \subseteq C$ and every closed line segment in $C$ with a relative interior point in $L$ has both endpoints in $L$. Let $K$ be an arbitrary subset of $\mathbb{R}^{J}$. Then, the convex cone generated by $K$ is a subset of $\mathbb{R}^{J}$ containing zero and all those vectors which can be represented as a linear combination with positive weights of finitely many points in $K$. The convex cone generated by the empty set consists of the zero vector alone. 
Given a matrix $A \in \mathcal{A}_{+}$, let $\Theta_{+}$and $\Theta_{+}^{i}$, be closed convex cones defined by

$$
\Theta_{+}=\left\{\theta \in \mathbb{R}^{J} \mid A \theta \in \mathbb{R}_{+}^{S}\right\}, \quad \Theta_{+}^{i}=\left\{\theta \in \Theta_{+} \mid \theta_{j}=0 \text { if } j \in \mathcal{J} \mathcal{J}\right\}
$$

Thus, the set $\Theta_{+}$consist of all those portfolios that give a hypothetical omnipresent investor nonnegative payoff in every state. There are portfolios that can potentially violate the no-arbitrage conditions $\mathrm{NAC}^{\mathrm{m}}$. The set $\Theta_{+}^{i}$ selects only those portfolios in $\Theta_{+}$that are feasible to agent $i$. These are portfolios that can potentially violate the no-arbitrage conditions NAC ${ }^{i}$.

Let $T$ denote the set of all those vectors $\theta \in \Theta_{+}$with $\|\theta\|=1$ such that the half-line emanating from the origin and passing through $\theta$ is an extreme ray of the cone $\Theta_{+}$. We refer to the set $T$ as the set of fundamental portfolios.

Lemma 4.1. Let the matrix $A \in \mathcal{A}_{+}$have rank $J$. Then $\Theta_{+}$is the convex cone generated by $T$. The set $T$ is non-empty.

Proof. The fact that the set $T$ generates the cone $\Theta_{+}$is an immediate implication of Corollary 18.5.2 of Rockafellar (1997). Since $A$ belongs to $\mathcal{A}_{+}$, there is a non-zero vector in $\Theta_{+}$. It follows that the set $T$ is non-empty, for the convex cone generated by the empty set consists of the zero vector alone.

Now consider the following conditions:

(C1) $\cap_{i \in \mathcal{I}} Q^{i} \subseteq Q^{\mathrm{m}}$

(C2) $\Theta_{+} \subseteq \sum_{i \in \mathcal{I}^{\prime}} \Theta_{+}^{i}$;

(C3) $T \subseteq \cup_{i \in \mathcal{I}} \Theta_{+}^{i}$.

The first of these says that if none of the restricted investors $1, \ldots, I$ has an arbitrage opportunity, then neither the hypothetical omniscient agent has. Condition (C3) will play a crucial role in the rest of the proof. It says that each fundamental portfolio is feasible to some investor $i \in \mathcal{I}$. Condition (C2) can be interpreted as saying that each portfolio that gives an omniscient agent a non-negative return in every state is jointly feasible to the restricted investors in the market, in the sense that each such portfolio can be written as a sum of individual portfolios, where each individual portfolio is feasible to the respective investor and gives him a non-negative return in every state.

Lemma 4.2. Let $A \in \mathcal{A}_{+}$have rank $J$. Then the conditions $(C 1),(C 2)$, and (C3) are equivalent.

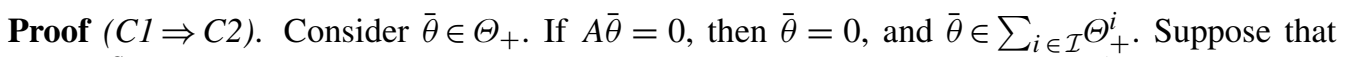
$A \bar{\theta} \in \mathbb{R}_{+}^{S} \backslash\{0\}$. By condition (C1), the inequality $0<q \bar{\theta}$ holds for all $q \in \cap_{i \in \mathcal{I}} Q^{i}$. That is, $0<q \bar{\theta}$ holds for all $(q, \pi) \in \mathbb{R}^{J} \times \mathbb{R}^{S I}$ satisfying

$$
q_{j}=\sum_{s \in \mathcal{S}} \pi_{s}^{i} A_{s}^{j}, \quad \text { for all } i \in \mathcal{I}, \quad j \in \mathcal{J}^{i}, \quad 0<\pi_{s}^{i}, \quad \text { for all } i \in \mathcal{I}, \quad s \in \mathcal{S}
$$

It follows that the inequality $0 \leq q \bar{\theta}$ holds for all $(q, \pi) \in \mathbb{R}^{J} \times \mathbb{R}^{S I}$ satisfying

$$
q_{j}=\sum_{s \in \mathcal{S}} \pi_{s}^{i} A_{s}^{j}, \quad \text { for all } i \in \mathcal{I}, \quad j \in \mathcal{J}, \quad 0 \leq \pi_{s}^{i}, \quad \text { for all } i \in \mathcal{I}, \quad s \in \mathcal{S}
$$

To see this, let $(q, \pi) \in \mathbb{R}^{J} \times \mathbb{R}^{S I}$ be such that the conditions in (2) are satisfied. Given an $\epsilon>0$, the pair $(q(\epsilon), \pi(\epsilon))$ satisfies the conditions in (1), where $\pi_{s}^{i}(\varepsilon)=\pi_{s}^{i}+\varepsilon, s \in \mathcal{S}$, and $q_{j}(\varepsilon)=$ $q_{j}+\varepsilon \sum_{s \in \mathcal{S}} A_{s}^{j}$. Therefore, $0<q(\varepsilon) \bar{\theta}$. Letting $\epsilon \downarrow 0$, we obtain $0 \leq q \bar{\theta}$, as desired. 
Now, we make use of Farkas' Lemma, see Rockafellar (1997, Corollary 22.3.1): for all $i \in \mathcal{I}$, $j \in \mathcal{J}^{i}$ and $s \in \mathcal{S}$ there exist numbers $\theta_{j}^{i}$ and $\mu_{s}^{i} \geq 0$ such that

$$
\sum_{j \in \mathcal{J}^{i}} A_{s}^{j} \theta_{j}^{i}-\mu_{s}^{i}=0, \quad \text { for all } i \in \mathcal{I}, \quad s \in \mathcal{S}, \quad \sum_{\left\{i \in \mathcal{I} \mid j \in \mathcal{J}^{\prime}\right\}} \theta_{j}^{i}=\bar{\theta}_{j}, \quad \text { for all } j \in \mathcal{J}
$$

Define $\theta_{j}^{i}$ to be zero for all $i \in \mathcal{I}$ and $j \in \mathcal{J} \mathcal{J}^{i}$ and let $\theta^{i}=\left(\theta_{1}^{i}, \ldots, \theta_{j}^{i}\right)$. Then, $\theta^{i} \in \Theta_{+}^{i}$ and $\sum_{i \in \mathcal{I}} \theta^{i}=\bar{\theta}$. Thus, $\bar{\theta} \in \sum_{i \in \mathcal{I}^{\Theta}} \Theta_{+}$.

$(\mathrm{C} 2 \Rightarrow \mathrm{C} 1)$ Given $q \in \cap_{i \in \mathcal{I}} Q^{i}$ we must show that $q \in Q^{\mathrm{m}}$. Consider $\theta \in \mathbb{R}^{J}$ be such that $A \theta \in \mathbb{R}_{+}^{S} \backslash\{0\}$. By condition (C2), there exist $\theta^{i} \in \Theta_{+}^{i}$ for $i \in \mathcal{I}$ such that $\sum_{i \in \mathcal{I}} \theta^{i}=\theta$. Moreover, there is $i_{0} \in \mathcal{I}$ with $A \theta^{i_{0}} \in \mathbb{R}_{+}^{S} \backslash\{0\}$. As $q \in Q^{i}$ for all $i \in \mathcal{I}$, we must have $0 \leq q \theta^{i}$ for all $i \in \mathcal{I}$ and $0<q \theta^{i_{0}}$. It follows that $0<q \theta$. Thus, $q \in Q^{\mathrm{m}}$.

$(\mathrm{C} 2 \Rightarrow \mathrm{C} 3)$ Let $\theta \in T$. As $\theta$ is an element of $\Theta_{+}$, condition (C2) implies that there are $\theta^{i} \in \Theta_{+}^{i}$ such that $\sum_{i \in \mathcal{I}} \theta^{i}=\theta$. As $\theta$ is a non-zero vector, there is $i_{0} \in \mathcal{I}$ such that $\theta^{i_{0}}$ is a non-zero vector. Observe that the line segment with endpoints $2 \theta^{i_{0}}$ and $2 \sum_{i \in \mathcal{I} \backslash\left\{i_{0}\right\}} \theta^{i}$ contains the vector $\theta$ in its relative interior. Since $\theta$ belongs to an extreme ray of the cone $\Theta_{+}$, both endpoints $2 \theta^{i_{0}}$ and $2 \sum_{i \in \mathcal{I} \backslash\left\{i_{0}\right\}} \theta^{i}$ belong to the same extreme ray. Therefore, there exists a positive number $t$ such that $2 \theta^{i_{0}}=t \theta$. This implies that $\theta$ is an element of $\Theta_{+}^{i_{0}}$.

$\left(\mathrm{C} 3 \Rightarrow \mathrm{C} 2\right.$ ) By Lemma 4.1, $\Theta_{+}$is the convex cone generated by $T$. By condition (C3), it is contained in the convex cone generated by $\cup_{i \in \mathcal{I}} \Theta_{+}^{i}$. Clearly, the convex cone generated by $\cup_{i \in \mathcal{I}} \Theta_{+}^{i}$ is equal to $\sum_{i \in \mathcal{I}} \Theta_{+}^{i}$.

For each $\theta \in \Theta_{+}$with $\|\theta\|=1$, let $\mathcal{S}(\theta)=\left\{s \in \mathcal{S} \mid A_{s} \theta=0\right\}$. Denote by codim $(\theta)$ the codimension of the linear subspace of $\mathbb{R}^{J}$ spanned by the vectors $A_{s}, s \in \mathcal{S}(\theta)$. The codimension of the linear subspace spanned by the vectors $A_{s}, s \in \mathcal{S}(\theta)$, together with vector $\theta$ equals $\operatorname{codim}(\theta)-1$. Moreover, observe that $\operatorname{codim}(\theta) \geq 1$. The next lemma shows that portfolios in $T$ are characterized by the property that $\operatorname{codim}(\theta)=1$. The intuition behind this result is that otherwise an appropriately chosen portfolio could both be added and subtracted from $\theta$ without leaving $\Theta_{+}$. This contradicts $\theta$ being a portfolio in $T$.

Lemma 4.3. Let $A \in \mathcal{A}_{+}$with rank $J$ and $\theta \in \Theta_{+}$with $\|\theta\|=1$ be given. Then $\theta \in T$ if and only if $\operatorname{codim}(\theta)=1$.

Proof. Let $L$ denote a half-line emanating from the origin and passing through the point $\theta$.

Suppose that $\operatorname{codim}(\theta)>1$. Then, the codimension of the linear space spanned by the vectors $A_{s}, s \in \mathcal{S}(\theta)$, together with vector $\theta$ is non-zero. Hence, there exists a vector $\xi \in \mathbb{R}^{J} \backslash\{0\}$ such that $A_{s} \xi=0$ for all $s \in \mathcal{S}(\theta)$ and $\theta \xi=0$. As $A_{s} \theta>0$ for all $s \in \mathcal{S} \backslash \mathcal{S}(\theta)$, there is an $\varepsilon>0$ such that $A_{s}(\theta+t \xi)>0$ for all $t \in[-\varepsilon, \varepsilon]$ and $s \in \mathcal{S} \backslash \mathcal{S}(\theta)$. Thus, the closed line segment with endpoints $(\theta-\varepsilon \xi)$ and $(\theta+\varepsilon \xi)$ lies entirely in $\Theta_{+}$and contains vector $\theta$ in its relative interior. However, neither of its endpoints belongs to $L$. Therefore, $L$ is not an extreme ray of $\Theta_{+}$, and $\theta$ is not an element of the set $T$.

Suppose that $\operatorname{codim}(\theta)=1$. Let $\theta^{\prime}$ and $\theta^{\prime \prime}$ be two points in $\Theta_{+}$such that $\lambda \theta^{\prime}+(1-\lambda) \theta^{\prime \prime}=t \theta$ for some $\lambda \in(0,1)$ and $t \geq 0$. We must show that $\theta^{\prime}$ and $\theta^{\prime \prime}$ both belong to $L$. Indeed, $A_{s} \theta^{\prime} \geq 0$ and $A_{s} \theta^{\prime \prime} \geq 0$ for all $s \in \mathcal{S}$. If $s \in \mathcal{S}(\theta)$, then $A_{s} \theta=0$, and therefore $A_{s} \theta^{\prime}=A_{s} \theta^{\prime \prime}=0$. Thus, all three vectors $\theta, \theta^{\prime}$, and $\theta^{\prime \prime}$ belong to a linear subspace orthogonal to the span of the vectors $A_{s}, s \in \mathcal{S}(\theta)$. As the dimension of this linear subspace is equal to 1 , there are real numbers $t^{\prime}$ and $t^{\prime \prime}$ such that $\theta^{\prime}=t^{\prime} \theta$ and $\theta^{\prime \prime}=t^{\prime \prime} \theta$. If the number $t^{\prime}$ were negative, $\theta^{\prime}$ would be a non-zero vector such that 
Table 1

The elements of the set $T$

\begin{tabular}{llllr}
\hline & Element 1 & Element 2 & Element 3 & Element 4 \\
\hline$j=1$ & 0 & $1 / \sqrt{2}$ & $-1 / \sqrt{5}$ & 0 \\
$j=2$ & $1 / \sqrt{2}$ & 0 & $2 / \sqrt{5}$ & $-1 \sqrt{5}$ \\
$j=3$ & $-1 / \sqrt{2}$ & $-1 \sqrt{2}$ & 0 & $2 \sqrt{5}$ \\
\hline
\end{tabular}

$0 \leq A \theta^{\prime}=t^{\prime} A \theta \leq 0$. This would contradict the assumption that $A$ has $\operatorname{rank} J$. Therefore, $t^{\prime} \geq 0$, and therefore $\theta^{\prime} \in L$. It follows similarly that $\theta^{\prime \prime} \in L$.

Corollary 4.1. Let $A \in \mathcal{A}_{+}$with rank $J$ and $\theta \in T$ be given. Then the set $\mathcal{S}(\theta)$ consists of at least $J-1$ distinct elements.

The following example illustrates the set $T$.

Example 4.1. Suppose that $I=3, S=4, J=3, \mathcal{J}=\mathcal{J}\{i\}$ for $i \in \mathcal{I}$, and

$$
A=\left[\begin{array}{lll}
1 & 1 & 1 \\
2 & 1 & 1 \\
1 & 2 & 1 \\
4 & 2 & 1
\end{array}\right]
$$

Observe that $A$ is an element of the set $\mathcal{A}_{+}$with rank $J$. Moreover, the matrix $A$ is in general position: each $(3 \times 3)$-dimensional submatrix of $A$ is non-singular. The set $T$ consists of the four elements reported in Table 1.

Elements 1 and 4 of the set $T$ belong to $\Theta_{+}^{1}$, element 2 belongs to $\Theta_{+}^{2}$, and element 3 belongs to $\Theta_{+}^{3}$. Since $T \subseteq \cup_{i \in \mathcal{I}} \Theta_{+}^{i}$, it follows by Lemma 4.2 that the sets $Q^{\mathrm{m}}$ and $\cap_{i \in \mathcal{I}} Q^{i}$ coincide.

Lemma 4.4. Suppose that $\mathcal{J} \neq \mathcal{J}$ for all $i \in \mathcal{I}$. Then there exists an open subset $\mathcal{A}^{\prime}$ of $\mathcal{A}$ with $\mathcal{A} \backslash \mathcal{A}^{\prime}$ having Lebesgue measure zero such that for all $A \in \mathcal{A}^{\prime} \cap \mathcal{A}_{+}, T \cap \Theta_{+}^{i}=\emptyset$ for all $i \in \mathcal{I}$.

Proof. For every $j \in \mathcal{J}$ and for every subset $M$ of $\mathcal{S}$ with cardinality $J-1$, define the function $F_{j M} \mathcal{A} \times \mathbb{R}^{J} \rightarrow \mathbb{R}^{J+1}$ as follows:

$$
F_{j M}(A, \theta)=\left[\begin{array}{c}
A_{s} \theta, s \in M \\
\theta \cdot \theta-1 \\
\theta_{j}
\end{array}\right]
$$

Define the sets $\mathcal{A}_{j M}$ as

$$
\mathcal{A}_{j M}=\left\{A \in \mathcal{A} \mid \text { there is no } \theta \in \mathbb{R}^{J} \text { such that } F_{j M}(A, \theta)=0\right\}
$$

To see that $\mathcal{A}_{j M}$ is open, let $A_{(n)}$ be the sequence of matrices in $\mathcal{A} \backslash \mathcal{A}_{j M}$ converging to some $A \in \mathcal{A}$. Then, there exists a sequence $\theta_{(n)}$ in $\mathbb{R}^{J}$ such that $F_{j M}\left(A_{(n)}, \theta_{(n)}\right)=0$ for all $n$. Since the sequence $\theta_{(n)}$ is bounded, it has a convergent subsequence converging to some $\theta \in \mathbb{R}^{J}$. Hence, $F_{j M}(A, \theta)=0$, and the matrix $A$ belongs to the complement of the set $\mathcal{A}_{j M}$.

The partial derivatives of the function $F_{j M}$ with respect to $\theta$ and $A_{s}, s \in M$, are represented in Table 2. For simplicity we take $M$ equal to $\{1, \ldots, J-1\}$. It is easy to see that for all $(A, \theta) \in F_{j M}^{-1}(0)$ 
Table 2

Partial derivatives of the function $F_{j M}, M=\{1, \ldots, J-1\}$

\begin{tabular}{llllll}
\hline & $\theta$ & $A_{1}$ & $A_{2}$ & $\cdots$ & $A_{J-1}$ \\
\hline$A_{1} \theta$ & $A_{1}$ & $\theta$ & 0 & $\cdots$ & 0 \\
$A_{2} \theta$ & $A_{2}$ & 0 & $\theta$ & $\cdots$ & 0 \\
$\vdots$ & $\vdots$ & $\vdots$ & $\vdots$ & $\ddots$ & $\vdots$ \\
$A_{J-1} \theta$ & $A_{J-1}$ & 0 & 0 & $\cdots$ & 0 \\
$\theta \cdot \theta-1$ & $2 \theta$ & 0 & 0 & $\cdots$ & 0 \\
$\theta_{j}$ & $e$ & 0 & 0 & $\cdots$ & 0 \\
\hline
\end{tabular}

The symbol $e$ is a $J$-dimensional row-vector such that $e_{l}=0$ for all $l \in \mathcal{J}\{j\}$ and $e_{j}=1$.

the matrix of the partial derivatives has full row rank. That is, $F_{j M}$ is transversal to zero. The Transversality Theorem implies that the complement of the set $\mathcal{A}_{j M}$ has Lebesgue measure zero.

Finally, define $\mathcal{A}^{\prime}$ as the set of matrices with rank $J$ in the intersection of all sets $\mathcal{A}_{j M}$. Then, $\mathcal{A}^{\prime}$ is open and its complement has Lebesgue measure zero.

Let $A \in \mathcal{A}^{\prime} \cap \mathcal{A}_{+}$and $\theta \in T$. Suppose that $\theta \in \Theta_{+}^{i}$ for some $i \in \mathcal{I}$. Then, $\theta_{j}=0$ for every $j \in \mathcal{J} \mathcal{J}$. Corollary 4.1 implies that there is a subset $M$ of the set $\mathcal{S}$ with cardinality $J-1$ such that $A_{s} \theta=0$ for all $s \in M$. Therefore, $F_{j M}(A, \theta)=0$ for every $j \in \mathcal{N} \mathcal{J}$, a contradiction to $A \in \mathcal{A}^{\prime}$. Thus, we have proved that $T \cap \Theta_{+}^{i}=\emptyset$ for all $i \in \mathcal{I}$ and $A \in \mathcal{A}^{\prime} \cap \mathcal{A}_{+}$.

Proof of Theorem 4.1. Define $\mathcal{A}_{+}^{*}$ to be the set of matrices with rank $J$ in the intersection of the sets $\mathcal{A}_{+}$and $\mathcal{A}^{\prime}$. As both the set of matrices in $\mathcal{A}$ with rank $J$ and the set $\mathcal{A}^{\prime}$ are open in $\mathcal{A}, \mathcal{A}_{+}^{*}$ is open in $\mathcal{A}_{+}$. Since both the set of matrices in $\mathcal{A}$ with rank $J$ and the set $\mathcal{A}^{\prime}$ have full Lebesgue measure, the set $\mathcal{A}_{+} \backslash \mathcal{A}_{+}^{*}$ has Lebesgue measure zero. For all $A \in \mathcal{A}_{+}^{*}$ the set $T$ is non-empty, whereas its intersection with the collection of cones $\Theta_{+}^{i}$ is empty. By Lemma 4.2, $\cap_{i \in \mathcal{I}} Q^{i} \neq Q^{\mathrm{m}}$.

\section{Acknowledgement}

The author would like to thank the Netherlands Organisation for Scientific Research (NWO) for financial support.

\section{References}

Basak, S., Croitoru, B., 2000. Equilibrium mispricing in a capital market with portfolio constraints. Review of Financial Studies 13, 715-748.

Polemarchakis, H.M., Siconolfi, P., 1997. Generic existence of competitive equilibria with restricted participation. Journal of Mathematical Economics 28, 289-311.

Radner, R., Rothschild, M., 1975. On the allocation of effort. Journal of Economic Theory 10, 358-376.

Rockafellar, R.T., 1997. Convex Analysis. Princeton University Press.

Sharpe, W.F., Alexander, G.J., 1990. Investments. Prentice Hall, Englewood, NJ.

Shleifer, A., 2000. Inefficient Markets-An Introduction to Behavioral Finance. Oxford University Press.

Siconolfi, P., 1988. Equilibrium with asymmetric constraints on portfolio holdings and incomplete financial markets. In: Gori, F., Geronazzo, L., Galeotti, M. (Eds.), Nonlinear Dynamics in Economics and the Social Sciences, Lecture Notes in Economics and Mathematical Systems, vol. 399. Springer-Verlag, Berlin, pp. 271-292.

Van Zandt, T., 1999. Real-time decentralized information processing as a model of organizations with boundedly rational agents. Review of Economic Studies 66, 633-658.

Vayanos, D., 2003. The decentralization of information processing in the presence of interactions. Review of Economic Studies 70, 667-695. 